\title{
Diagnosis of a ferroresonance type through visualisation
}

\author{
Łukasz Majka ${ }^{1, *}$, Maciej Klimas ${ }^{1}$ \\ ${ }^{1}$ Silesian University of Technology, Institute of Electrical Engineering and Computer Science, Akademicka 10, 44-100 Gliwice, Poland
}

\begin{abstract}
The paper is focused on presenting the possible enhancements in visualisation of the ferroresonance phenomenon. The investigations have been performed with the usage of overcurrent/overvoltage responses of a ferroresonance circuit. The waveforms have been measured and recorded in a ferroresonant test circuit. Phase-plane/-space graphs analysed in this paper are a visual display of certain type characteristics for the time dependent system of nonlinear differential equations. The application of Poincare maps is also mentioned in the paper.
\end{abstract}

\section{Ferroresonance phenomenon}

The proper tools for the analysis or prediction of an oscillating phenomenon like ferroresonance may be found in advanced computation methods, i.e. a numerical integration method $[1,2]$. Numerical implementations of the time-domain techniques are useful in analyses of power networks which are especially vulnerable to ferroresonance occurrence [3]. The phase plane and space visualization with the use of Poincaré maps allows to assess and classify a type of ferroresonance phenomenon induced in a circuit. It is critical for choosing the correct mathematical approach [4].

\section{Circuit measurements}

This test circuit is equipped with a regulated voltage source, a resistor, a nonlinear coil and a capacitor. Through an adjustment of the source voltage level one can intentionally induce a ferroresonance phenomenon in the circuit. The FeD system [5] was used to acquire the voltages and current waveforms of all circuit components (Fig. 1.) and in further computations. During the measurements the ferromagnetic core coil has reached its saturation region conditions.

a)

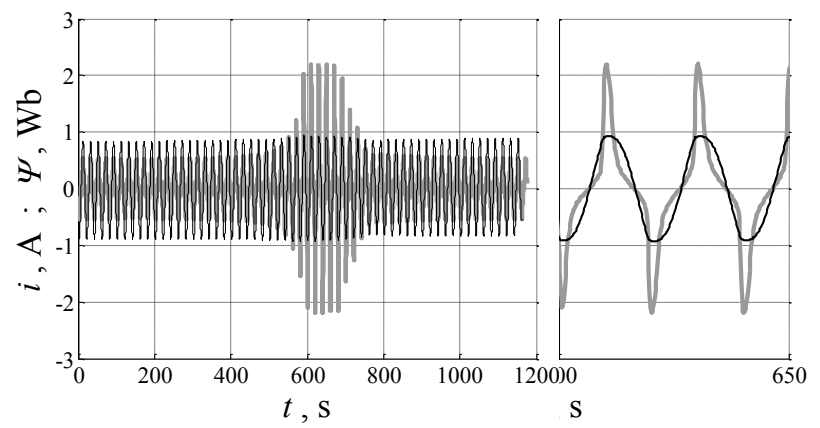

b)

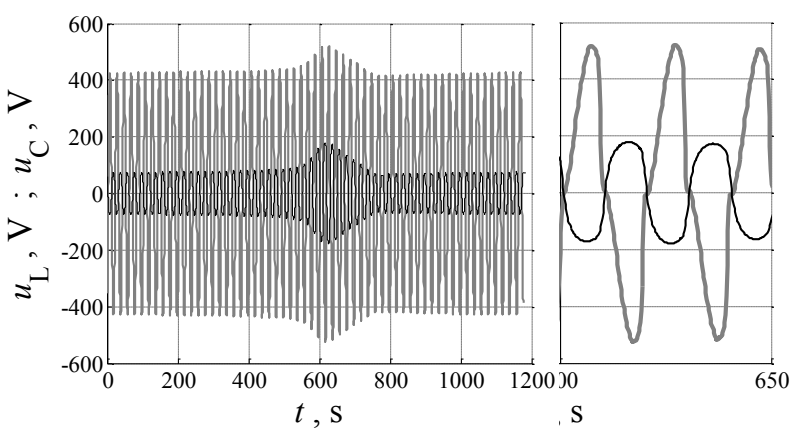

Fig. 1. Measured waveforms of circuit current (gray) and computed nonlinear coil magnetic flux (black) (a) and nonlinear coil (gray) and linear capacitor (black) measured voltage waveforms (b) during ferroresonance (the scale is common for both quantities).

The flux linkage, a quantity not measurable directly, was obtained on the basis of its definition as a time integral of the voltage across the coil [5].

\section{Iron core coil examination}

The nonlinear coil is a crucial element of each ferroresonant circuit. The shape of a hysteresis curve significantly influences the ferroresonance phenomenon $[6,7]$. Diagnostic of the test circuit coil was performed over a full range of supply voltage.

\section{Visualisation of ferroresonance}

Among recognized tools dedicated to study the nonlinear and dynamic systems, a diagnostic approach to the ferroresonance is optimal with $2 \mathrm{D} / 3 \mathrm{D}$ phase plane analyses supported by Poincaré maps [5, 8]. Such solution with upgrades was proposed in this paper to correctly classify the ferroresonance type that has occurred in the test circuit.

\footnotetext{
* Corresponding author: lukasz.majka@polsl.pl
} 
The set of differential equations depending on assumed mathematical model of nonlinear coil is used to describe a nonlinear and dynamical test circuit:

$$
\left\{\begin{array}{c}
\frac{\mathrm{d} \Psi}{\mathrm{d} t}=f_{1}\left(\Psi, u_{\mathrm{C}}, t\right) \\
\frac{\mathrm{d} u_{\mathrm{C}}}{\mathrm{d} t}=f_{2}\left(\Psi, u_{\mathrm{C}}, t\right) \\
\frac{\mathrm{d} t}{\mathrm{~d} t}=1
\end{array}\right.
$$

Such system of differential equations is solved numerically. The time development of state variables $u_{\mathrm{C}}$ and $\Psi$ forms trajectories displaying ferroresonance occurrence (Fig. 2).

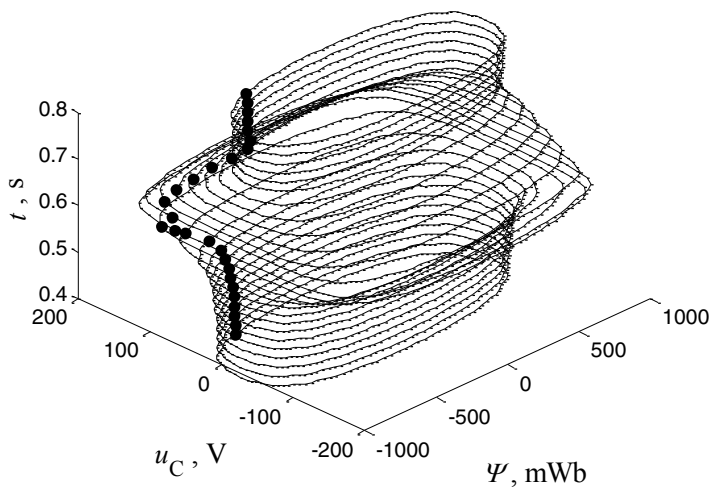

Fig. 2. Phase trajectory of the investigated system (where ferroresonance is observed).

The presence of a periodically driven voltage source and independent variables in the discussed system allows to generate another type of a three dimensional space. This space is an ordinary cylindrical coordinate system generated by rotating $\Psi-u_{\mathrm{C}}$ plane around a central vertical axis. The angular coordinate $\omega_{0} t$ represents the stage of the source waveform in its period T (Fig. 3).

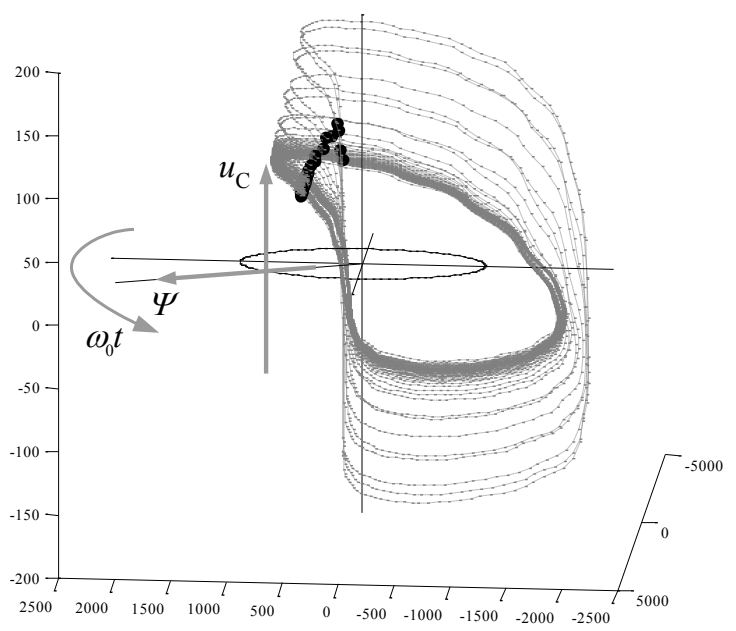

Fig. 3. Three dimensional representation of trajectory generated through the rotating $\Psi-u_{\mathrm{C}}$ plane for an examined ferroresonance transient state.

The intersections of the trajectories with a plane at an arbitrarily selected angle are recorded. These intersections allow to generate what is called a Poincaré map (Fig. 4).

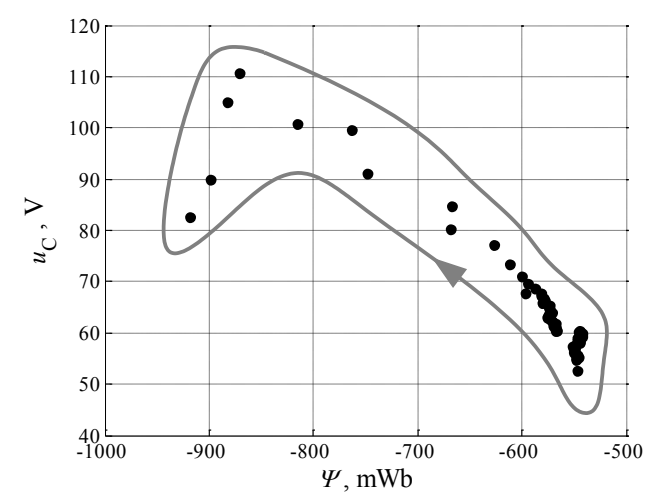

Fig. 4. Poincare map for the studied trajectory.

A record of the intersection points (forming the Poincare map) along with the trajectory in a 3-dimensional space is applied back to Fig. 2.

The analysis of the features of an obtained Poincare map can lead to the determination of the type of ferroresonance, which in a practical system could lead to the identification of the source of the phenomenon. A reliable ferroresonance mode classification has been given in the study [4]. The occurrence studied in this paper is one that can be classified as a quasi-periodic mode. The closed path of points on the Poincare maps can be observed (Fig. 4). Several paths could be observed when imaging Poincare maps over a larger time interval (e.g. from waveforms as in [4]).

\section{Conclusions}

The series ferroresonance circuit has been studied. The obtained waveforms allow to illustrate 3D and 2D plots of the system phase trajectories. Further analysis of phase trajectories made it possible to obtain a Poincare map, and this in turn allowed to determine the type of the occurring ferroresonance.

\section{References}

1. S. Seker, T.C. Akinci, S. Taskin, El. En. 94, 2 (2012)

2. M. Sowa, Bull. Pol. Ac.: Tech. 66, 4 (2018)

3. D.A.N. Jacobson, IEEE Pow. En. Soc. Gen. Meet. (2003)

4. Ł. Majka, Bull. Pol. Ac.: Tech. 66, 4 (2018)

5. Ł. Majka, M. Klimas, El. En. 101 (2019), doi: 10.1007/s00202-019-00761-5

6. A. Rezaei-Zare, R. Iravani, M. Sanaye-Pasand, IEEE Trans. Pow. Deliv. 24, 1 (2009)

7. K. Chwastek, A.P.S. Baghel, B. Sai Ram, B. Borowik, L. Daniel, S.V. Kulkarni, J. Appl. Phys. 51, 14 (2018)

8. J.A. Corea-Araujo, F. Gonzalez-Molina, J.A. Martinez, J.A. Barrado-Rodrigo, L. GuaschPesquer, IEEE Trans. Pow. Deliv. 29, 6 (2014) 